\title{
Software Defined Radio-based General Modulation/Demodulation Platform for Enhancing Undergraduate Communication and Networking Curricula
}

\section{Dr. Zhiping Zhang, Wright State University}

Zhiping Zhang received his B.S. degree in electrical engineering from Nankai University, Tianjin, China, in 2001, M.S. and Ph.D. degrees in intelligence science from Peking University, Beijing, China, in 2004 and 2011 respectively. From 2011 to 2013, he was a Postdoctoral Research Fellow at the Department of Computer Science and Technology, Peking University. Since 2013, he has served as a research faculty member and co-director of Broadband, Mobile and Wireless Networking Laboratory at the Department of Electrical Engineering of Wright State University.

Dr. Zhiqiang Wu, Wright State University

Dr. Zhiqiang Wu received his BS from Beijing University of Posts and Telecommunications in 1993, MS from Peking University in 1996, and PhD from Colorado State University in 2002, all in electrical engineering. He has worked at West Virginia University Institute of Technology as assistant professor from 2003 to 2005. He joined Wright State University in 2005 and currently serves as full professor. Dr. $\mathrm{Wu}$ is the author of national CDMA network management standard of China. He also co-authored one of the first books on multi-carrier transmission for wireless communication. He has published more than 100 papers in journals and conferences. He has served as Chair of Acoustic Communication Interest Group of IEEE Technical Committee on Multimedia Communications. His research has been supported by the National Science Foundation, Air Force Office of Scientific Research, Air Force Research Laboratory, Office of Naval Research, and NASA. His work on software defined radio implementation of cognitive radio won the Best Demo Award at IEEE Globecom 2010.

\section{Prof. Bin Wang, Wright State University}

Prof. Bin Wang earned his Ph.D. from the Ohio State University in 2000. He joined the Wright State University in September 2000, where he is currently full professor of computer science and engineering. His research interests include optical networks, real-time computing, mobile and wireless networks, cognitive radio networks, trust and information security, and semantic web. He is a recipient of the US Department of Energy Career Award. His research has been supported by US Department of Energy, National Science Foundation, Air Force Office of Scientific Research, Air Force Research Laboratories, Ohio Supercomputer Center, and the State of Ohio.

\section{Prof. Chi-Hao Cheng, Miami University}

Dr. Chi-Hao Cheng received the B.S. degree in control engineering from National Chiao Tung University, Taiwan in 1991, and the M.S. and Ph.D. degrees from The University of Texas at Austin in 1996 and 1998 respectively, both in Electrical and Computer Engineering. He is currently a professor in the Department of Electrical and Computer Engineering at Miami University, Ohio. His primary professional interests lie in signal processing algorithm development and its applications in numerous communications system and component development including wireless and optical communications systems. He is co-inventors of three US patents.

\section{Dr. Deng Cao, Central State University}

Dr. Deng Cao received his Ph.D in Computer Science from West Virginia University in 2013. He earned two master degrees in Statistics and Physics from West Virginia University, and his bachelor degree in Physics from Hunan Normal University in China. Dr. Cao joined Central State University in 2013 and currently serves as an assistant professor in the department of Mathematics and Computer Science. His research interests include advanced biometrics, computer vision, pattern recognition and machine learning. 


\title{
Software Defined Radio based General Modulation/Demodulation Platform for Enhancing Undergraduate Communication and Networking Curricula
}

\begin{abstract}
We are living in a world of wireless communication and networking and the advances of communication and networking have changed the world fundamentally. Communication and networking courses, especially wireless communication and networking courses, have become an integral part of the Electrical Engineering, Computer Science, and Computer Engineering curricula. Due to costly hardware needed for communication and networking teaching laboratories, many of these courses are taught without a laboratory. Additionally, such hardware based teaching labs lack the flexibility to evolve over time and adapt to different environments.
\end{abstract}

Supported by an NSF TUES type II grant, we have developed a software defined radio (SDR) based general modulation/demodulation platform for enhancing undergraduate communication and networking curricula. In our previous NSF funded CCLI project "Evolvable wireless laboratory design and implementation for enhancing undergraduate wireless engineering education", we have developed and demonstrated the first nationwide example of evolvable SDR based laboratories for three existing undergraduate courses. In this project, we are developing new lab components that can be adopted by multiple courses ranging from freshman year introductory course to senior year capstone design projects. Specifically, we have developed a SDR based general modulation/demodulation platform with a graphical user interface (GUI). This user-friendly GUI will allow students to adjust RF parameters such as carrier frequency and symbol rate. More importantly, this general modulation/demodulation platform is capable of transmitting many popular modulation schemes such as BPSK, QPSK, 8PSK, 16PSK, 16QAM, 64QAM. Additionally, students are able to observe the transmitted signal in both time and frequency, and observe the constellation of the modulation at both the transmitter and the receiver. Furthermore, this platform enables students to design their own new modulation constellations via the GUI. At the receiver side, the SDR based platform also provides students the functionality of demodulating the data symbols and bits, and compare them with the transmitted data symbols and bits to calculate the symbol error rate (SER) and the bit error rate (BER) under different channel conditions and signal to noise ratios (SNRs). By assigning constellation points in the signal space and evaluating the SER/BER performance of the constellation, students gain hands-on experiences on the digital modulation/demodulation in realistic wireless communication channels.

Through collaboration among the three participating institutions (Wright State University, Miami University (a mostly undergraduate serving institution), and Central State University (an HBCU)), the developed SDR based general modulation/demodulation platform will be integrated in undergraduate curricula of all three institutions. 


\section{Introduction}

Need and Motivation: We are living in a world of wireless communication and networking. More than $90 \%$ of the US population use wireless services such as cell phones and WiFi every day ${ }^{[1][2]}$. Communication and networking has become an integral part of the Electrical Engineering, Computer Science, and Computer Engineering curricula. Additionally, this explosion of wireless technology poses a new promising opportunity to attract new talent into the Science, Technology, Engineering, and Mathematics (STEM) programs and to revolutionize our undergraduate education ${ }^{[3]}$.

Electrical engineering and computer science are practical disciplines, and students learn by doing. Hands-on experience is an integral part of their learning. Students learn from equations and homework; they learn from computer simulations through Matlab and Labview; but nothing beats a real laboratory where they can get their hands dirty. However, many communication and networking courses are taught at different universities without a real hands-on lab. There is a simple reason behind this: many schools cannot afford such laboratories.

Traditionally, communication and networking laboratories are conducted using RF equipment such as radio frequency signal generators and spectrum analyzers, or custom-made/special laboratory equipment. Such equipment is always expensive and it is not feasible to purchase multiple sets of such equipment to accommodate an undergraduate teaching lab. For example, the TIMS (Telecommunications Instructional Modeling System) communication laboratory equipment ${ }^{[4]}$ used by most of the few universities (e.g., Auburn University ${ }^{[5]}$ and Georgia Tech ${ }^{[6]}$ ) offering laboratories in their curricula costs $\$ 100,000$ for one basic setup.

To solve this problem, we have developed and demonstrated the first nationwide example of evolvable software defined radio (SDR) ${ }^{[7]-[13]}$ based communication and networking laboratories Supported by an NSF CCLI type I project, we developed an affordable SDR based platform and multiple experiments on it to serve three undergraduate communication and networking courses ${ }^{[14][15]}$. Inspired by the success of the type I project, we have received support from an NSF TUES type II grant to extend and expand our effort to develop more teaching labs based on this SDR platform both vertically and horizontally. Specifically, we are designing and developing more lab modules to serve a wide range of courses from freshman year introduction courses to capstone senior design courses. Additionally, the three participating institutions of this project (Wright State University, Miami University (a mostly undergraduate serving institution), and Central State University (an HBCU)) are collaborating to deploy the lab modules developed at each individual institution across all campuses.

In this paper, we report a software defined radio based general modulation/demodulation platform with a graphical user interface (GUI) to give students hands-on experiences with digital modulation constellation and communication performance analysis. This user-friendly GUI will allow students to adjust RF parameters such as carrier frequency and symbol rate. More importantly, this general modulation/demodulation platform is capable of transmitting many popular modulation schemes such as BPSK, QPSK, 8PSK, 16PSK, 16QAM, 64QAM. 
Additionally, students are able to observe the transmitted signal in both time and frequency, and observe the constellation of the modulation at both the transmitter and the receiver. Furthermore, this platform enables students to design their own new modulation constellations via the GUI. At the receiver side, the SDR based platform also provides students the functionality of demodulating the data symbols and bits, and compare them with the transmitted data symbols and bits to calculate the symbol error rate (SER) and the bit error rate (BER) under different channel conditions and signal to noise ratios (SNRs).

\section{SDR based General Modulation/Demodulation Platform}

Digital modulation and demodulation is an important component of communication and networking curricula. Traditionally, labs on different modulations require different hardware. Employing SDR, we are now able to combine and integrate multiple digital modulation schemes together on one universal platform. This also allows students to see the connection between different digital modulations.

Figure 1 shows the SDR based general modulation/demodulation platform. As shown in Figure 1, we have developed a graphic user interface (GUI) to control various types of SDR hardware. Specifically, we have used the universal software radio peripheral (USRP) ${ }^{[12]}$ hardware by ETTUS and National Instruments because of its popularity in academia and its affordability. We have developed the platform on four different kinds of USRP boards: USRP1, USRP2, USRP N210, and USRP X300. We have also used arbitrary waveform generator, vector spectrum analyzer, wireless channel emulator and digital phosphor oscilloscope to validate and expedite the development of our lab modules.

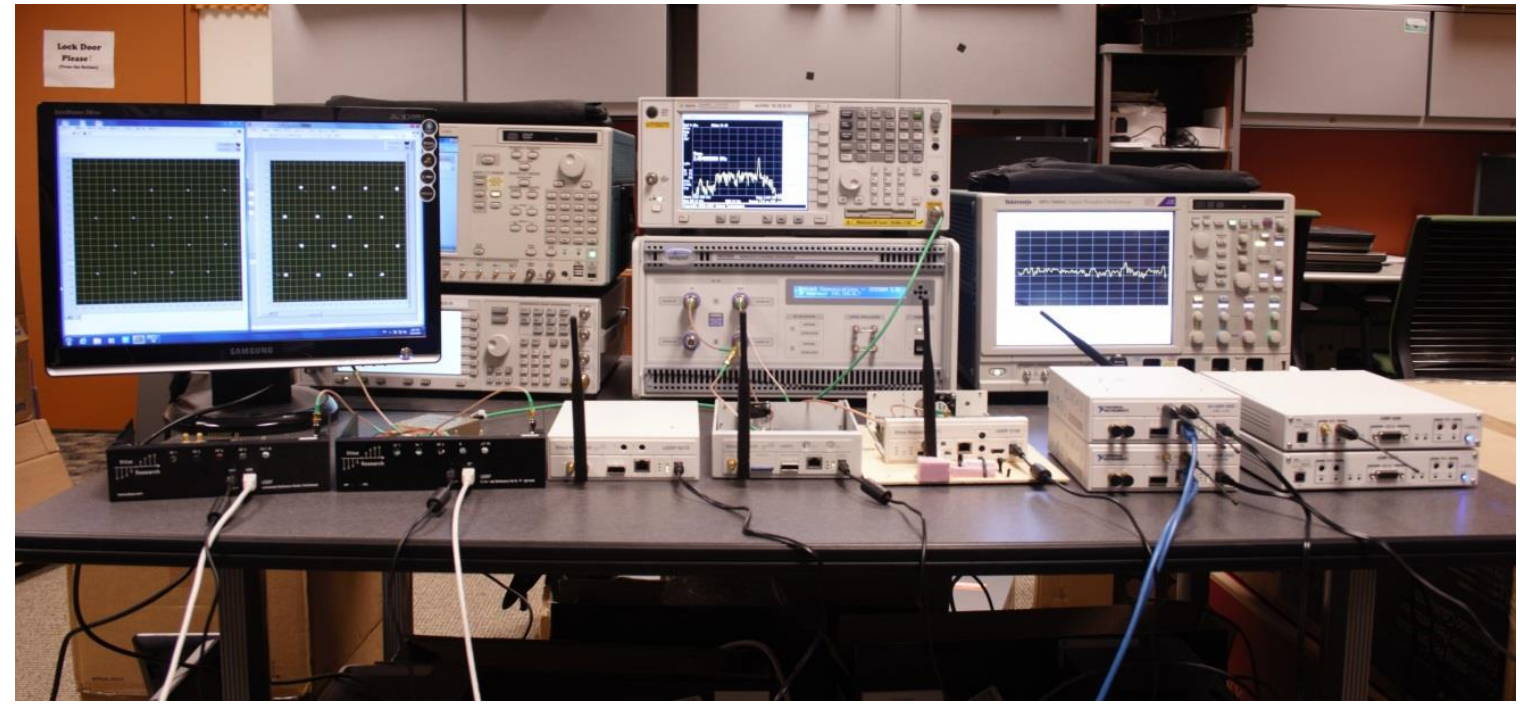

Figure 1. SDR based General Modulation/Demodulation Platform 
We have employed Labview and Matlab to develop the general modulation/demodulation platform. Figure 2 and Figure 3 illustrate the Labview block diagram of the transmitter and receiver of the general modulation/demodulation platform.

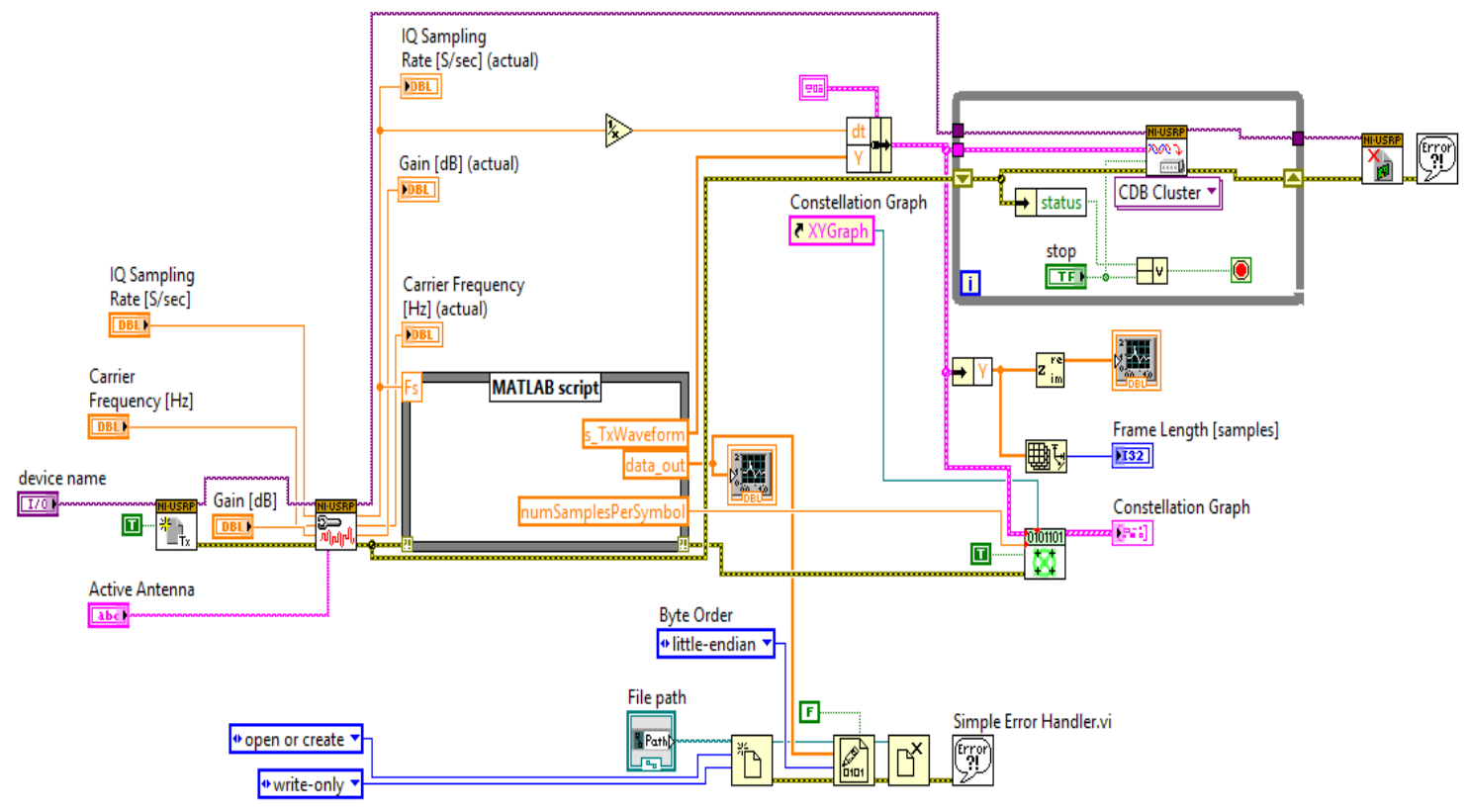

Figure 2. Transmitter of the SDR based General Modulation/Demodulation Platform

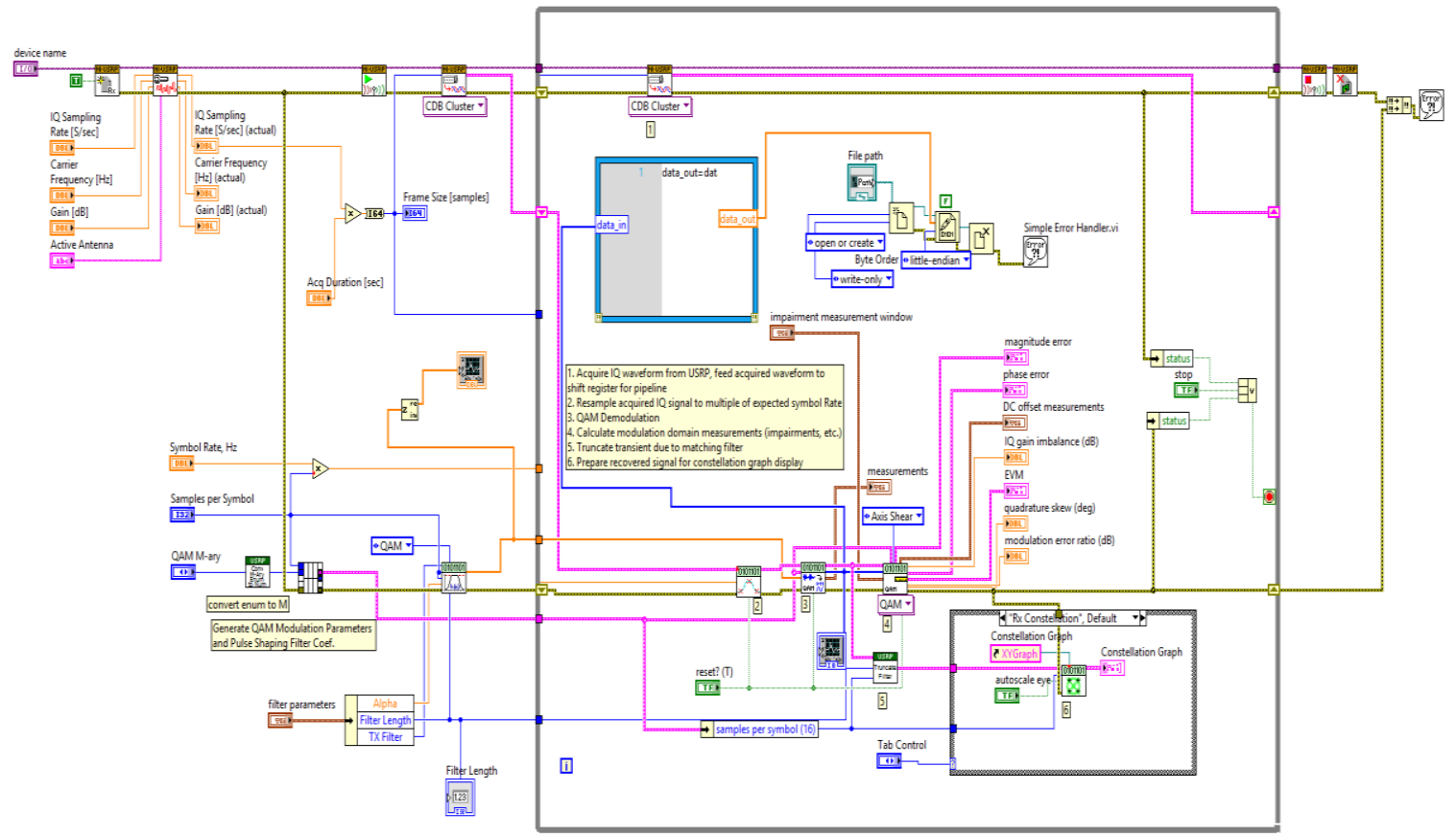

Figure 3. Receiver of the SDR based General Modulation/Demodulation Platform 
In both the transmitter and receiver block diagrams (shown in Figure 2 and Figure 3), there is a signal processing block implemented in Matlab for the digital modulation constellation. Students can read the Matlab script to see how the digital modulation constellation is determined. Additionally, students are able to modify the Matlab script to design their own digital modulation constellations.

Figure 4 shows the GUI of the transmitter side of the SDR based general modulation/demodulation platform. As shown in the figure, students can easily modify different RF parameters of the transmission such as carrier frequency and symbol rate. Three windows display the digital modulation constellation, the time domain transmitted signal, and the frequency spectrum of the transmitted signal.

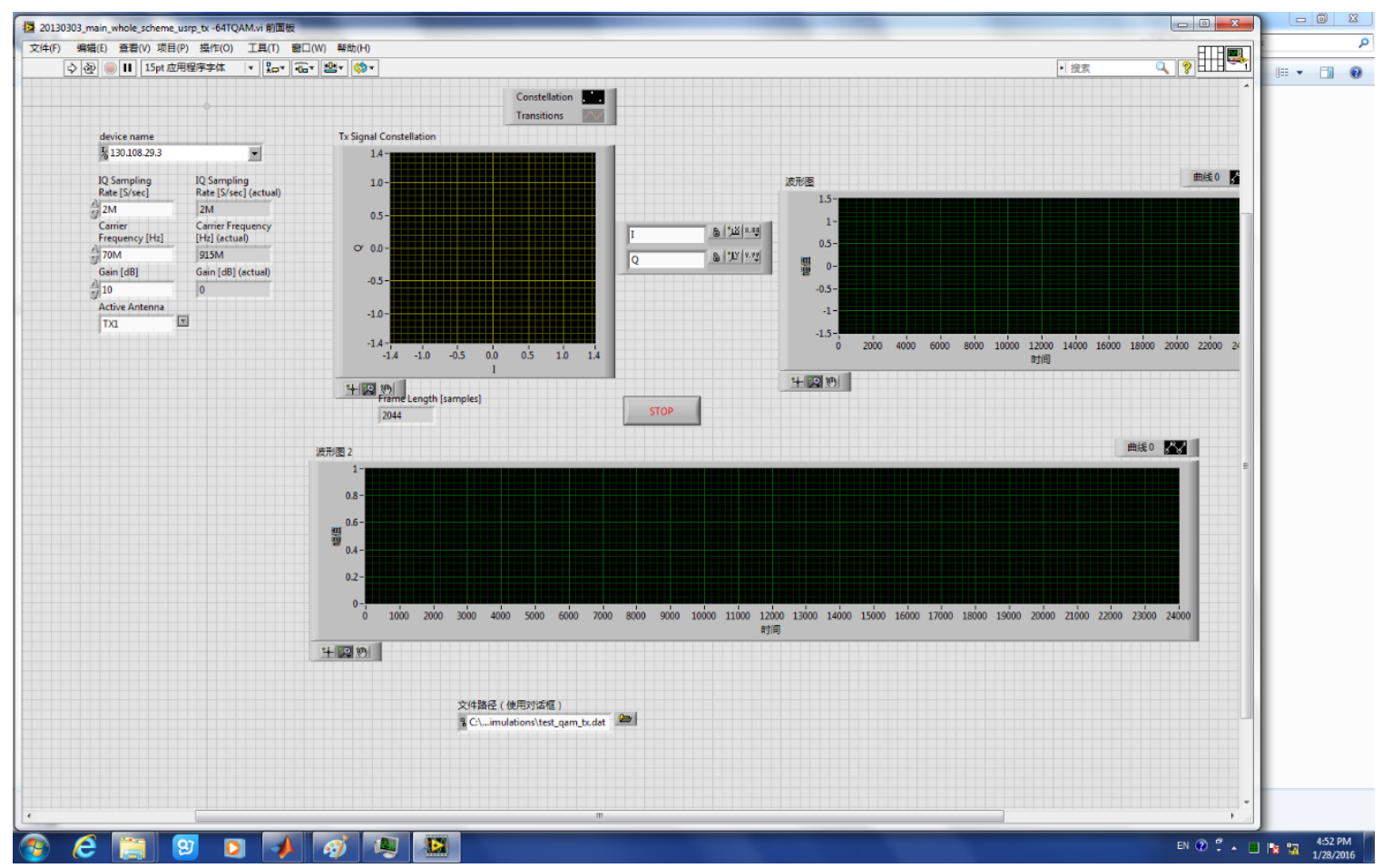

Figure 4. Graphic User Interface of Transmitter

Similarly, a GUI at the receiver side allows students to observe the received signal in both time domain and frequency domain, along with the received digital modulation constellation. Figure 5 shows the GUI at the receiver side. It is important to note that the receiver has to know the exact digital modulation constellation, carrier frequency and symbol rate determined at the transmitter side. 
The transmitter and receiver of the SDRs can be connected by an RF cable, or RF antennas. We have also developed a white noise generator in the platform. Using an RF cable to connect the transmitter and receiver and varying the noise power, we can observe the disruption of the digital modulation constellation at different signal to noise ratios.

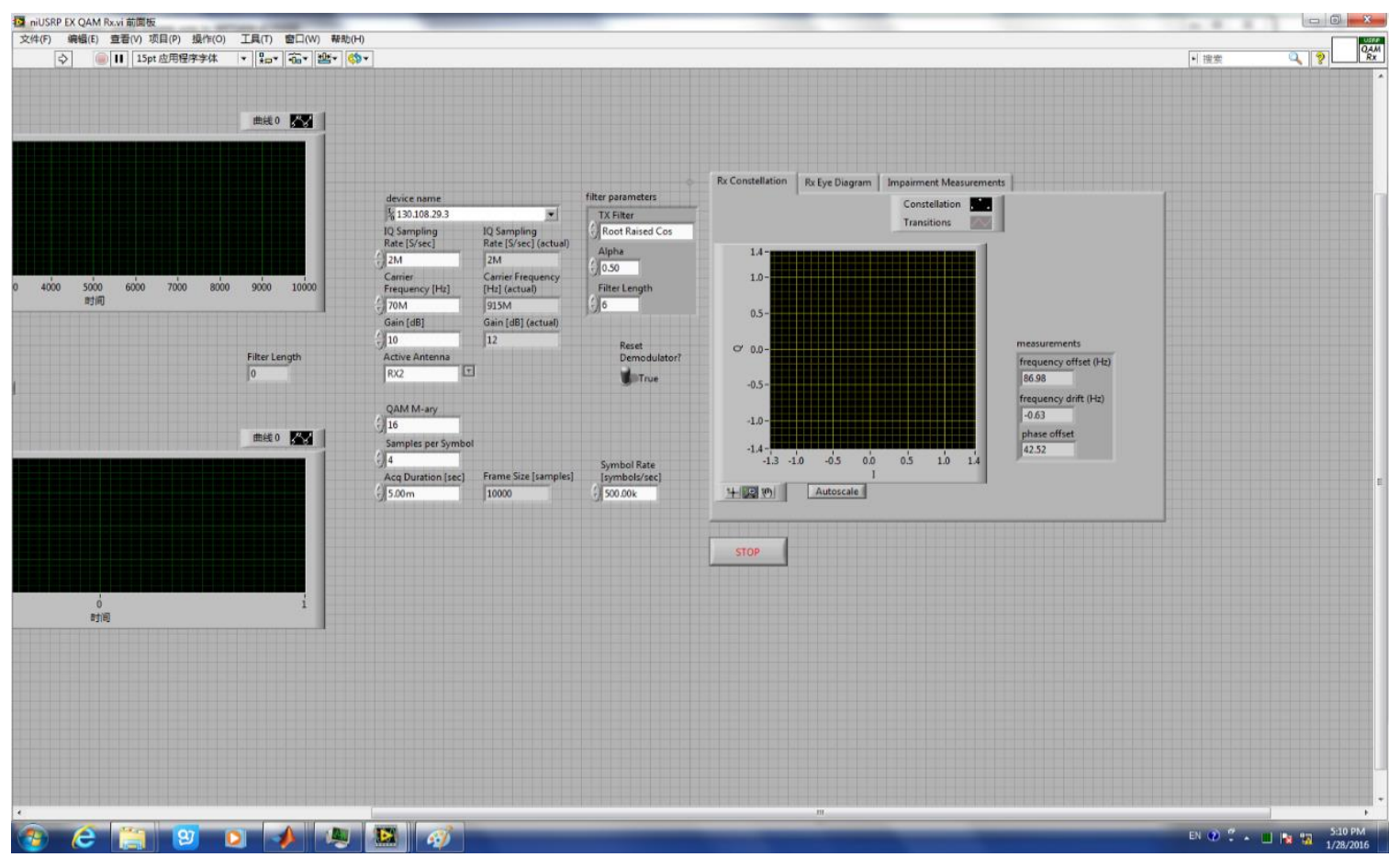

Figure 5. Graphic User Interface of Receiver

\section{Laboratory Examples}

Many different laboratories can be designed and developed using this general modulation/demodulation platform. The following Matlab script shows the modulation scheme of QPSK. We have included in the script many options of different digital modulations such as BPSK, QPSK, 8PSK, 16QAM, 64QAM etc. Students can change the desired digital modulation by simply uncommenting the few lines of Matlab code in the provided script. 


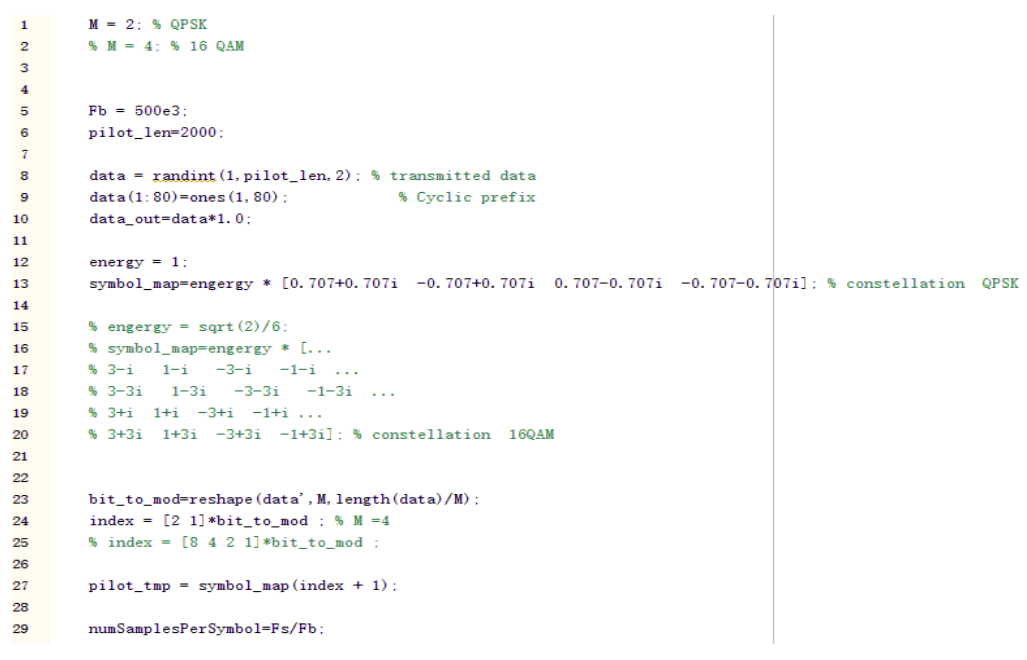

Figure 6. Matlab Script to Change Modulation Constellation

Figure 7 (a) and (b) show the transmitted QPSK constellation and received QPSK constellation from the SDR based general modulation/demodulation platform.

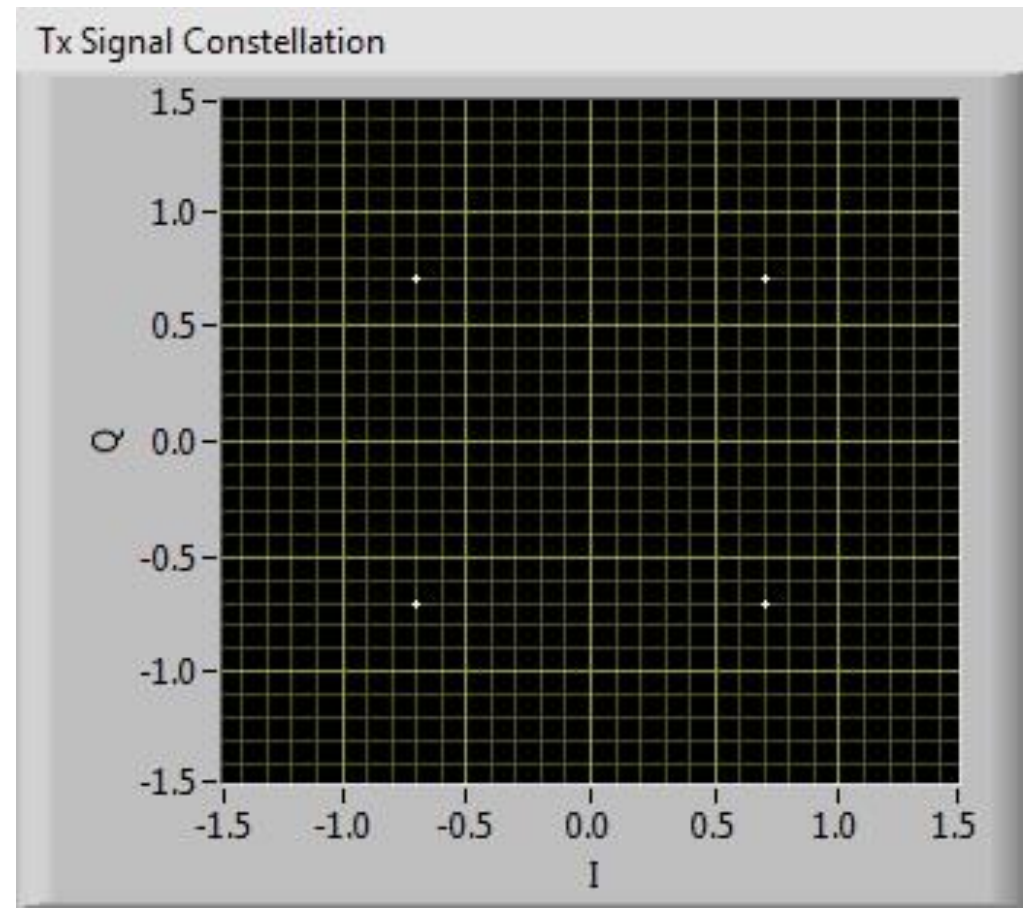

(a) Transmitted Constellation 


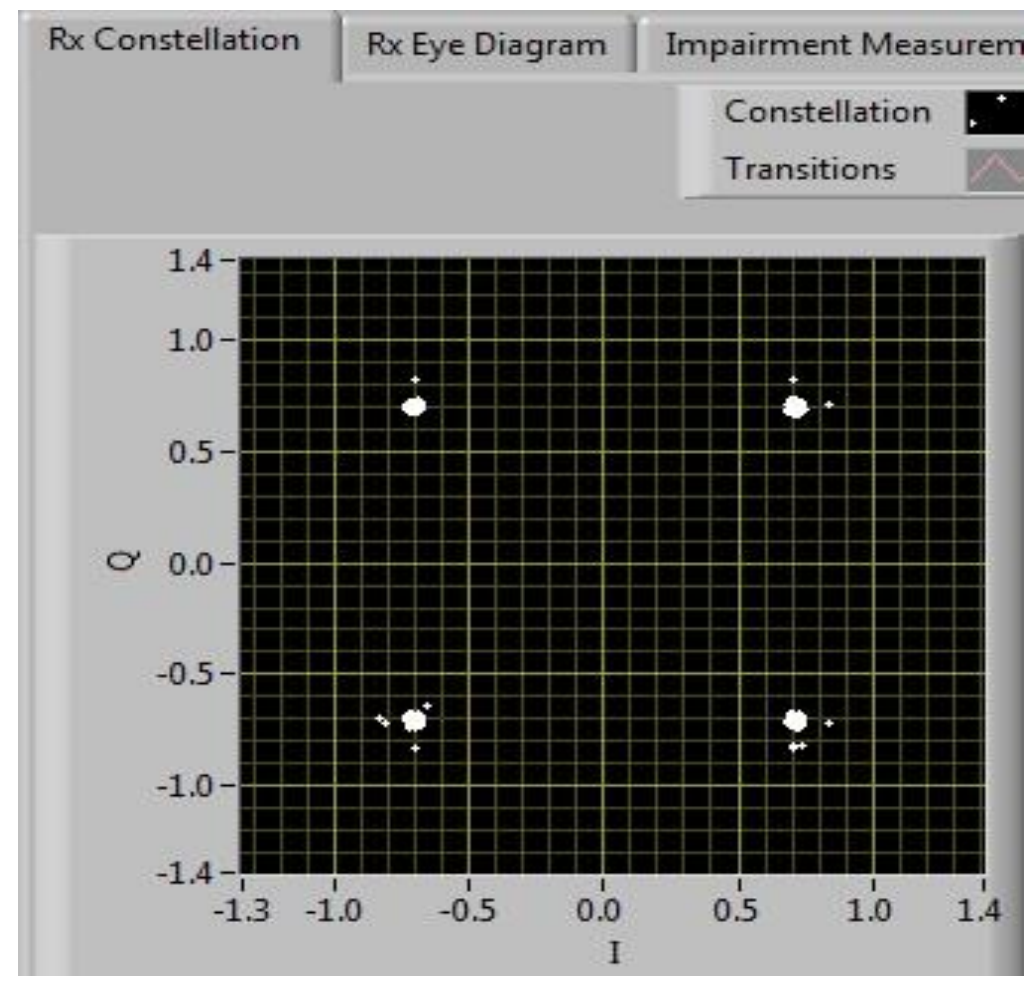

(b) Received Constellation

Figure 7. Transmitted and Received Digital Modulation Constellations of QPSK

Figure 8 shows another example of 16QAM.

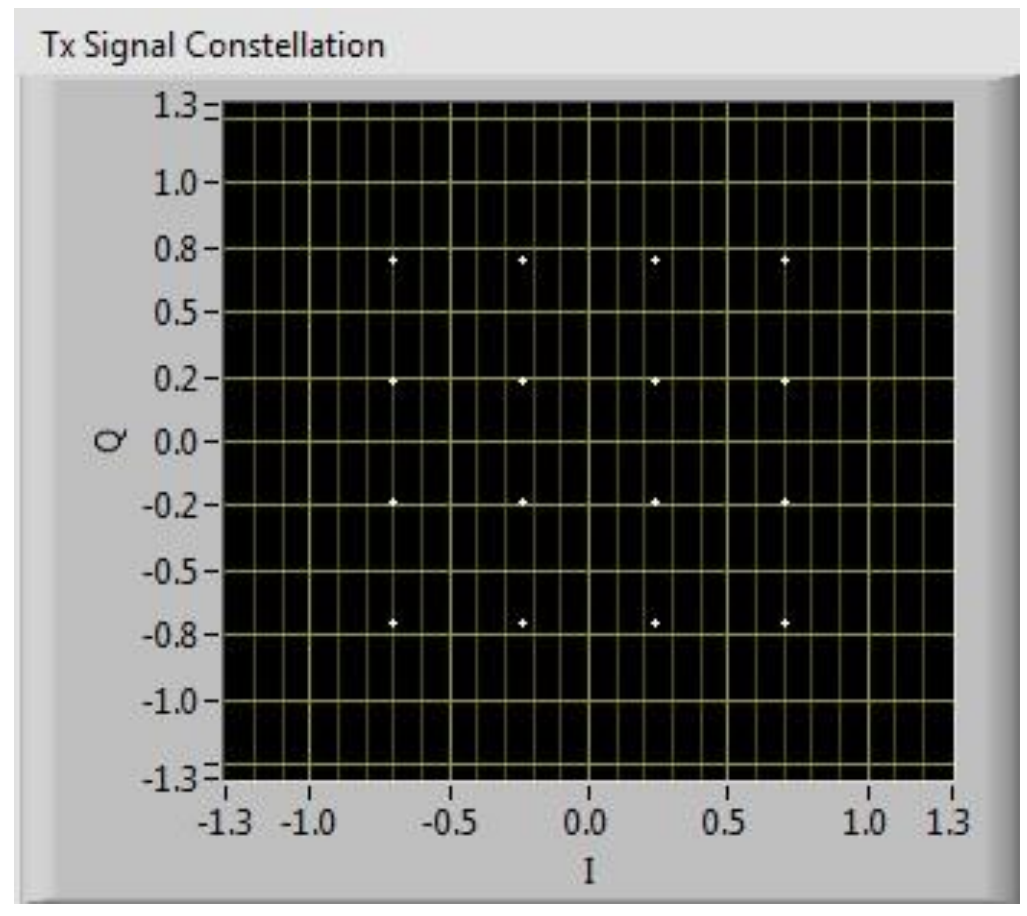

(a) Transmitted Constellation 


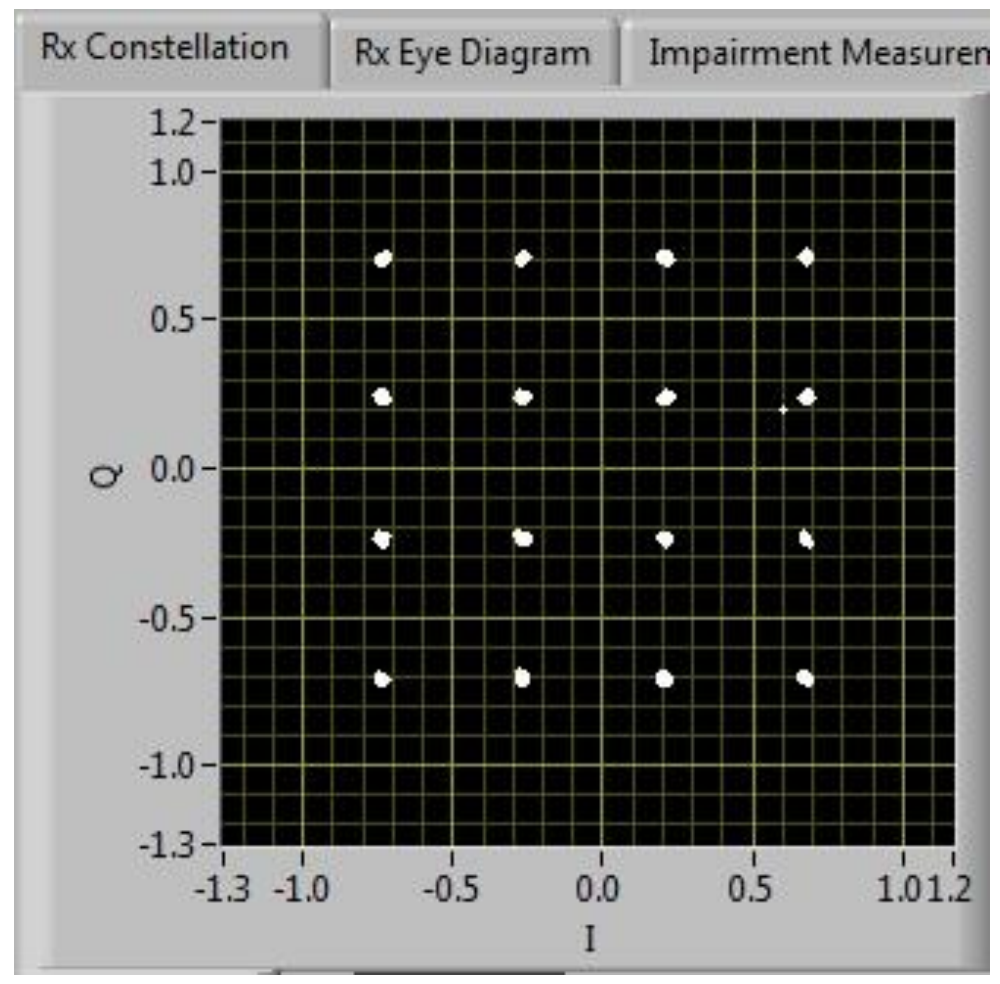

(b) Received Constellation

Figure 8. Transmitted and Received Digital Modulation Constellations of 16QAM

It is important and interesting to note that in addition to commonly used digital modulations, students can design and transmit irregular digital modulations or even their own digital modulations, simply by providing new constellation coordinates in the Matlab script. Figure 9 shows an example of the received constellation of an irregular 8PSK digital constellation. 


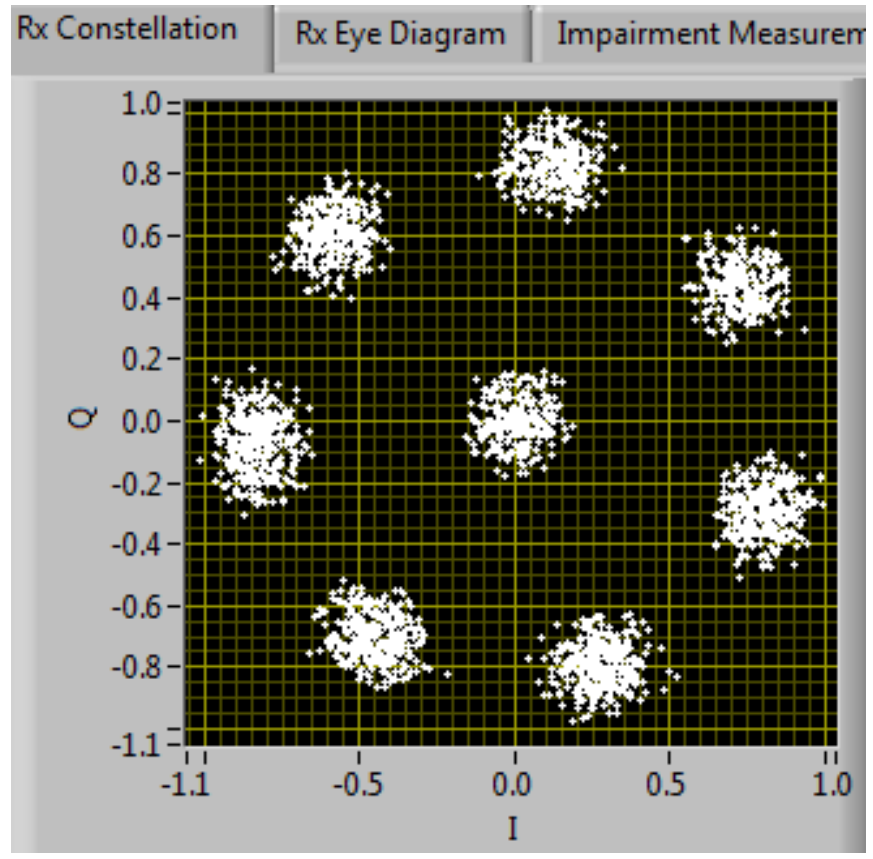

Figure 9. Received Digital Modulation Constellation of Irregular 8PSK

\section{Enhancing Curricula}

The developed SDR based general modulation/demodulation platform has become a very useful tool to enhance the communication and networking curricula at Wright State University, University of Miami, and Central State University. The platform is very user friendly through its GUI and its graphical display of the constellation. Labs built upon this platform have been adopted in multiple courses such as EE4210/6210 Digital Communication, EE4730/6730 Wireless Communication, EE7350 Wireless Communication Techniques, and EE8000 Advanced Electronic Warfare.

The platform provides students with an intuitive understanding of the digital modulation and demodulation. By adjusting RF parameters and designing their own modulations, students are excited and gain important hands-on experiences. During the course review, we have received universal positive feedback on the labs and the SDR based platform.

Due to the graphical manner of the digital modulation constellation, this platform is also a useful tool to attract high school students into STEM fields. We have shown this platform to multiple groups of visiting high school students at Wright State University and invariably students have been highly intrigued by the intuitive graphical display of advanced digital modulations. 


\section{Conclusions}

Supported by an NSF TUES type II grant, we have developed a software defined radio (SDR) based general modulation/demodulation platform for enhancing undergraduate communication and networking curricula. The SDR based platform allows students to observe the communication signals in time domain and frequency domain, as well as the digital modulation constellation at both the transmitter side and the receiver side. Students can easily adjust RF parameters and digital modulation schemes of the communication through a GUI. Additionally, students can design and implement their own modulation constellations. We have developed a series of laboratories on this platform and integrated them in multiple courses across three participating institutions.

\section{References:}

[1] U.S. Wireless Quick Facts, CTIA-The Wireless Association, http://www.ctia.org/advocacy/research/index.cfm/aid/10323

[2] IEEE 802.11 standard, http://standards.ieee.org/about/get/802/802.11.html

[3] Option in Wireless Engineering, College of Engineering and Computer Sciences, Wright State University, http://www.cs.wright.edu/cecs/academic-programs.shtml

[4] Telecommunications Instructional Modeling System, http://www.tims.com.au

[5] Auburn University Wireless Engineering Undergraduate Program, http://www.eng.auburn.edu/wireless/

[6] Georgia Institute of Technology, ECE 4602, http://www.ece.gatech.edu/academics/undergrad/course_schedule.pdf

[7] National Instruments Digital Communication Teaching Bundle, http://sine.ni.com/nips/cds/view/p/lang/en/nid/210087

[8] Software Defined Radio Forum, http://www.sdrforum.org

[9] M. Dillinger, K. Madani and N. Alonistioti, Software Defined Radio: Architectures, Systems and Functions, Wiley, 2003

[10] W. Tuttlebee, Software Defined Radio: Enabling Technologies, Wiley, 2002

[11] P. Kenington, RF and Baseband Techniques for Software Defined Radio, Artech House Publishers, 2005

[12] Universal Software Radio Peripheral, http://www.ettus.com/

[13] GNU Radio, http://gnuradio.org 
[14] B. Wang, Y. Pei and Z. Wu, "Software Radio Based Wireless Laboratory Design and Implementation for Enhancing Undergraduate Wireless Engineering Education," Proceedings of 2010 Annual ASEE Conference and Exposition, Louisville, KY June 2010.

[15] B. Guenther, B. Rahn, B. Wang and Z. Wu, "Real-Time Video Transmission from High Altitude Balloon: An Interdisciplinary Senior Design Project," Proceedings of 2010 Annual ASEE Conference and Exposition, Louisville, KY June 2010. 